\title{
Management of Intracranial Haemorrhage in Haemophilia
}

\author{
S. H. DAVIES,* M.B., CH.B., F.R.C.P.ED., PH.C. ; J. W. TURNER, † M.B., B.S., F.R.C.S.ED. \\ R. A. CUMMING, $\ddagger$ O.B.E., M.B., CH.B., F.C.PATH. ; F. J. GILLINGHAM, $\dagger$ M.B.E., M.B., B.S., F.R.C.S.ED., F.R.C.S. \\ R. H. GIRDWOOD,§ M.D., PH.D., F.R.C.P., F.R.C.P.ED., F.C.PATH.; ALEXIS DARG, $\ddagger$ A.I.M.L.T.
}

Brit. med. Э., 1966, 2, 1627-1630

The success of efforts to control bleeding episodes in patients with classical haemophilia (factor VIII deficiency) has increased steadily in Great Britain in the past two decades. During this period special haemophilia reference centres have been established, and better facilities provided for the transfusion of blood and its products. Meanwhile laboratory tests have been developed which enable a precise diagnosis of the disease to be made and treatment to be controlled scientifically and effectively. None the less, intracranial bleeding, whether spontaneous or traumatic in origin, has remained a dangerous complication of the disease, and Silverstein (1960) reported a mortality of $70 \%$ in haemophilioid states (factor VIII, IX, or XI deficiency) and Kerr (1964) reported an overall mortality of $33 \%$.

Marked improvement in both mortality and morbidity from this complication can now be expected as a result of the successful production of human and animal plasma protein fractions containing a high concentration of the antihaemophilic factor (factor VIII). The successful use of these products in the management of intracranial bleeding in haemophilic patients is reported in this paper.

\section{Clinical Material}

Five patients with classical haemophilia and proved associated intracranial bleeding, in all of whom adequate treatment of their haemophilia was possible, form the basis of this report. In the course of five years, four were admitted to the Royal Infirmary of Edinburgh and one to the Western General Hospital, Edinburgh. The four patients admitted to the Royal Infirmary represent $3.4 \%$ of the haemophilic admissions to this hospital for all forms of haemorrhage in the same period. This compares with an incidence elsewhere of $6.3 \%$ (Silverstein, 1960) and of about $7.6 \%$ (Kerr, 1964), though in some of the latter cases the diagnosis of intracranial bleeding was not fully substantiated.

The five patients were all diagnosed as suffering from classical haemophilia either on the present or a previous admission to hospital, and showed a plasma deficit under the conditions of the thromboplastin generation test (Biggs and Douglas, 1953) and a subnormal level of factor VIII in their plasma when assayed by the method of Biggs et al. (1955). In our experience, based on clinical and laboratory findings which correlate well, haemophiliacs can be classified as severely, moderately, or mildly affected. Those patients who are severely affected tend to bleed spontaneously and have a factor VIII plasma level of less than $1 \%$; their whole-blood clotting-time is usually markedly prolonged, though sometimes it is within normal limits. Moderately affected patients have between 1 and $10 \%$ of factor VIII in their plasma, and they bleed readily and prolongedly from minor trauma and also sometimes spontaneously. Mild cases have a plasma level of factor VIII

\footnotetext{
- Department of Haematology.

$t$ Department of Neurosurgery.

₹egional Blood Transfusion Service.

Department of Therapeutics.

The Royal Infirmary, Edinburgh.
}

of 10 to $50 \%$ and seldom bleed excessively except as a result of definite trauma.

Of the five patients in this report one was a severe haemophiliac, two were moderate, and two were mild. Three of them (one in each category) had a clear history of head injury, but in the other two there was no immediate history of trauma. All five patients had a positive family history of haemophilia.

The method of preparation of the human antihaemophilic products used has previously been reported (Cumming et al., 1965).

\section{Case 1}

A youth aged 19 with haemophilia of mild severity (factor VIII level $25 \%$ ) was admitted to hospital in September 1961. In December 1960 he had struck the right temporal region of his head in a minor road accident but had no immediate sequelae. However, two months later he developed right-sided Jacksonian epileptic fits, which were controlled by anticonvulsant therapy. In August 1961 he again injured his head in a fall at home, and soon after this he developed headaches and later nausea, vomiting, deterioration of memory, and diplopia. One month later he gradually became disorientated and had palsy of the right sixth cranial nerve with bilateral papilloedema. Spontaneous bleeding began from the gums and genitourinary tract.

On admission no fracture was found on skull $x$-ray examination. He was given a rapid intravenous infusion of 2 units of human antihaemophilic factor (A.H.F.) over a total period of two hours. Immediately before use each unit was reconstituted in $200 \mathrm{ml}$. of pyrogen-free sterile water for injection. The patient's plasma factor VIII level rose to $102 \%$. Lumbar puncture and lumbar air encephalography were then performed. The cerebrospinal fluid (C.S.F.) was xanthochromic and the air encephalogram showed moderate hydrocephalus. Left percutaneous common carotid angiography merely confirmed the hydrocephalus.

Daily lumbar puncture was done over the next 10 days to drain excess C.S.F. In order to cover these procedures and to ensure uninterrupted healing of the punctured carotid artery, it was thought advisable, in the early stages, to maintain a normal level of factor VIII in the plasma-that is, $60 \%$ or more. This was achieved in the first six days by six-hourly intravenous infusions of 1 unit of human A.H.F. given over a period of one hour; the intravenous drip was maintained in the interim by very slow normal saline infusion. The adequacy of the A.H.F. therapy was monitored by measuring plasma factor VIII levels immediately before and after each A.H.F. infusion for the first 24 hours and thereafter at least twice daily. For a further four days the patient was given $400 \mathrm{ml}$. of fresh frozen plasma over two hours every six hours. He made a complete recovery.

\section{Case 2}

A man aged 56 with mild haemophilia (factor VIII level 18\%) was admitted to hospital in November 1962. Five days before admission and with no known preceding injury he developed severe and progressive occipital headaches which radiated forwards and were followed by vomiting. There was no loss of consciousness. He showed marked neck stiffness and an equivocal left plantar reflex. $\mathrm{He}$ had benign essential hypertension (blood pressure 190/110 mm. $\mathrm{Hg}$ ) with grade II hypertensive retinopathy. His blood urea level 
was normal. Skull $x$-ray examination showed nothing abnormal, but E.E.G. showed some left-sided dysfunction. He was transfused with a total of 2 units of human A.H.F. over two hours, which raised his plasma factor VIII level to $70 \%$, and lumbar puncture was then performed. Sterile blood-stained C.S.F. with supernatant xanthochromia was obtained. Bilateral percutaneous common carotid angiography gave normal radiographs but the posterior cerebral circulation was not demonstrated. One unit of human A.H.F. was then given six-hourly over a period of one hour for the next four days and therapy was monitored by measurement of factor VIII in the patient's plasma, as in Case 1. Thereafter he was given $400 \mathrm{ml}$. of fresh frozen plasma over two hours every six hours for a further three days. He then made a steady and complete recovery.

\section{Case 3}

A boy aged 2 years with haemophilia of moderate severity (factor VIII level $8 \%$ ) was admitted to hospital in August 1964. Three days before admission he struck his head during play, and this caused a superficial bruise over the left parietal region. On the day of admission he vomited, and three hours after this was found unconscious, making no response to painful stimuli. The pupils were fixed, dilated, and unequal, the left larger than the right. $\mathrm{He}$ showed decerebrate movements. One unit of human A.H.F. was given intravenously in one hour and skull burr-holes were made. A large, tense left subdural haematoma was found and evacuated. Burr-hole exploration on the right side showed no clot. The patient's level of consciousness improved dramatically with restoration of purposive response, but he had a left hemiplegia and left oculomotor nerve paresis. Normal haemostasis was maintained for the next 10 days by the administration of 1 unit of human A.H.F. every six hours. His condition gradually improved and the neurological deficits disappeared. Serial echo-encephalography did not suggest any recurrence of bleeding.

\section{Case 4}

A man aged 26 with haemophilia of moderate severity (factor VIII level $6 \%$ ) was admitted to hospital in January 1964 . Four days before admission, and without known injury, he developed frontal headaches, with sneezing, rhinorrhoea, postnasal drip, and pain over the left maxillary antrum. He had no treatment and continued at work as a clerk. On the day of admission he involuntarily dropped his pencil from his dominant right hand and then developed nominal and expressive dysphasia. Very slight neck stiffness was present. Neurological testing showed minimal impairment of fine finger movement, absent cutaneous reflexes, and an equivocal plantar reflex, all confined to the right side. His blood white cell count was normal. The neurological deficits rapidly increased in severity. E.E.G. showed a localized abnormality in the left frontoparietal area, and $x$-ray examination of his antra revealed a hazy opacity of the left side. Nasal swabs proved to be bacteriologically sterile.

He was given two units of human A.H.F. in two hours, and his plasma factor VIII level rose to $62 \%$. After a further two units in two hours the level was $100 \%$ (Fig. 1). During this time a lumbar puncture yielded blood-stained but sterile C.S.F. with supernatant xanthochromia. Human A.H.F. therapy was continued for a further six days on the basis of one unit every six hours, and

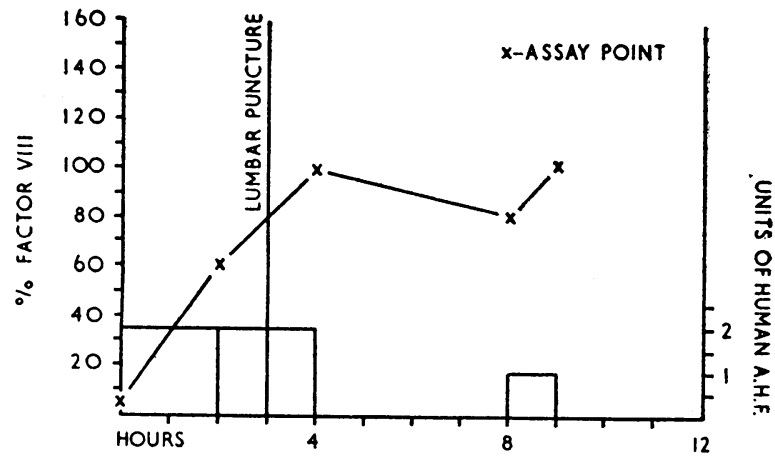

Fig. 1.-Case 4. Factor VIII plasma levels after transfusion of human A.H.F. concentrate (1 unit is prepared from 1,350 ml. of fresh A.C.D. plasma.) frequent measurements of his plasma factor VIII levels were all normal. Despite this he progressively deteriorated, became aphasic, and developed a dense right-sided hemiparesis and right homonymous hemianopia. Four days after admission percutaneous left common carotid angiography showed narrowing of vessels beyond the siphon with some bowing of the anterior cerebral artery, but the lesion was thought to be too small to warrant neurosurgical intervention. Later that day he developed right-sided Jacksonian epilepsy culminating in status epilepticus, which was controlled by an intravenous thiopentone sodium drip over the next 24 hours. He then steadily improved. The human A.H.F. was eventually replaced by $400 \mathrm{ml}$. of fresh frozen plasma six-hourly for four days, and he made a complete clinical recovery.

\section{Case 5}

A man aged 21 with severe haemophilia (factor VIII level less than $1 \%$ ) was admitted to hospital in March 1961. On the day of admission he had been thrown from a motorcycle and rendered temporarily unconscious, sustaining minor lacerations of the chin and left eyebrow, and rapidly developing a left orbital haematoma. $\mathrm{He}$ was found to be blind in the left eye, and skull $x$-ray films showed an extensive comminuted fracture in the left anterior cranial fossa with distortion of the left optic foramen.

$\mathrm{He}$ was given 2 units of human A.H.F. and $400 \mathrm{ml}$. of fresh frozen plasma in the next four hours when his plasma factor VIII level was $62 \%$. Minor lacerations were treated. C.S.F. rhinorrhoea was noted. Further human A.H.F. was given initially as 1 unit every four hours for 48 hours and thereafter as 1 unit, together with $400 \mathrm{ml}$. of fresh frozen plasma, every six hours. The adequacy of the therapy was controlled by frequent plasma factor VIII assays. On the day after admission he had a left epistaxis, which was controlled by nasal packing. However, each time the pack was removed the epistaxis soon recurred with increasing severity and was preceded by severe left frontal headache. He then developed impaired movement of his left eye associated with increasing proptosis; the pupil reacted consensually to light. Percutaneous left common carotid angiography confirmed the tentative clinical diagnosis of traumatic aneurysm of the left internal carotid artery at the origin of the ophthalmic artery. Under local anaesthesia the left common carotid artery was ligated after it had been shown that there was an adequate collateral cerebral circulation. Adequate A.H.F. therapy was continued for a further eight days and there were no epistaxes. On the 12 th postoperative day he had a small left epistaxis, followed two days later by severe bleeding, and the usual A.H.F. regimen was resumed. At the same time, under local anaesthesia, the left internal carotid artery was exposed, and, after angiography had shown the aneurysmal sac to be unchanged in size, the artery was first clamped, and, when no neurological deficit developed, ligated. Human A.H.F. therapy was discontinued after a further uneventful 10 days but profuse left epistaxis recurred two days later.

Under cover of porcine A.H.F. (Fig. 2) (human A.H.F. stocks were exhausted) angiography demonstrated the aneurysmal sac to be still present ; craniotomy was performed and the sac isolated, opened, cleared of clot, and packed with muscle. Immediately after the operation the patient showed a transient right-sided hemiparesis, which cleared rapidly. Porcine A.H.F. therapy was continued. On the 12 th postoperative day there was a purulent discharge from his

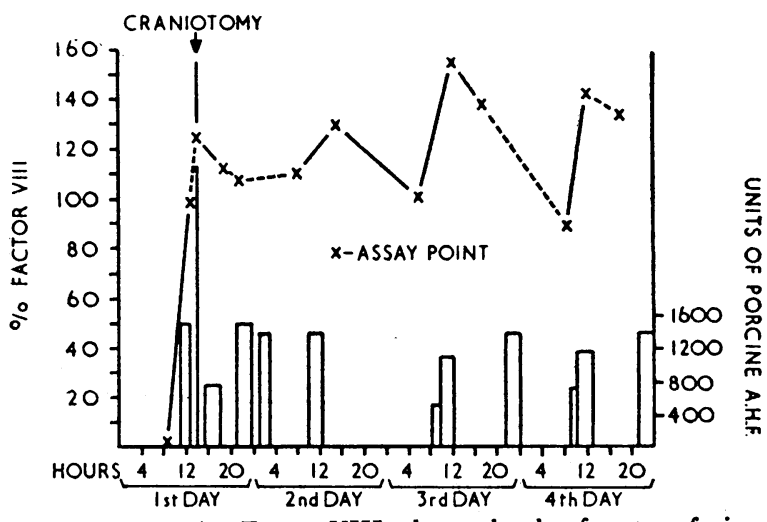

FIg. 2.-Case 5. Factor VIII plasma levels after transfusion of porcine A.H.F. ( 1 unit is approximately equivalent to $4 \mathrm{ml}$. of normal plasma). 
left nostril, which gave a growth of $\beta$-haemolytic and anaerobic streptococci. Appropriate oral antibiotic therapy was begun. Two days later he had a brisk left-sided epistaxis. Percutaneous right common carotid angiography did not show the aneurysmal sac, but air was seen within the skull, and displacement of the anterior and middle cerebral vessels indicated the presence of a left frontal lesion, thought to be an abscess. His condition deteriorated rapidly, with fever, tachycardia, left epistaxis, and confusion, and he died on the 16th postcraniotomy day.

At necropsy a left frontal-lobe abscess was found with subdural pus, meningitis, and ventriculitis. No communication was observed between the left internal carotid artery or its branches and the site of the false aneurysm, which had been satisfactorily excluded from the normal circulation. Death was thought to be due to infection and secondary haemorrhage from granulation tissue.

The amount of human blood products used in each case solely to cover the procedure of the percutaneous carotid angiography is shown in the Table.

Preangiographic Plasma Factor VIII Levels Achieved with Intravenous Preangiographic Plasma Factor VIII Levels Achieved
Human A.H.F. Concentrate Therapy

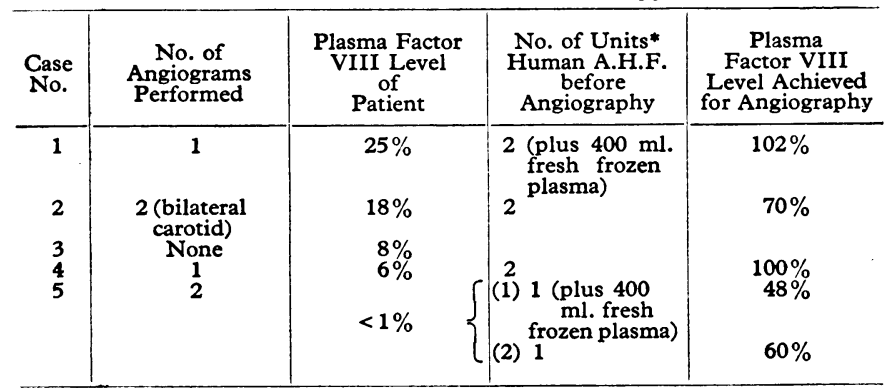

- 1 unit human A.H.F. is prepared from 1,350 ml. fresh A.C.D. human plasma.

\section{Discussion}

In order to establish the diagnosis of intracranial haemorrhage in a suspected or known haemophiliac, and to exclude a causal lesion requiring immediate operative intervention, it is first essential to confirm, as a matter of urgency, that the patient has a subnormal plasma level of factor VIII and to determine its exact value. Prompt and adequate intravenous A.H.F. therapy, controlled by frequent laboratory assays, will then ensure continuous normal haemostasis in the patient and enable the diagnostic procedures of lumbar puncture, air encephalography, percutaneous carotid angiography, etc., to be undertaken as necessary steps in the investigation of the intracranial bleeding.

A.H.F. is unstable in solution in vitro. It has a short halflife in vivo of about 12 hours (Biggs, 1957); this is probably because in the intact human organism the haemostatic mechanism is in a state of dynamic equilibrium (Astrup, 1956). Consumption of clotting-factors (including factor VIII) is therefore increased where, in the presence of overt trauma, locally enhanced haemostasis is needed to form and maintain a viable clot, and this is particularly so where clot formation is inadequate and blood loss persists. In normal people the plasma level of factor VIII ranges from 50 to $200 \%$ (Biggs and Macfarlane, 1962). To achieve such factor levels in a severe haemophiliac so that his haemostatic mechanism is brought to normality, and to maintain it until healing is well established, presents a major problem of treatment. Firstly, it is necessary to obtain a factor VIII preparation of such potency that circulatory overload does not occur during its administration, and, secondly, it is essential to have adequate supplies of it in order to sustain treatment.

Fresh plasma, while fairly easily obtainable, has such a low content of factor VIII that it is impossible to achieve and maintain safe haemostasis with it because of the large volume needed. Human A.H.F. concentrate can be a satisfactory alternative preparation (Cumming et al., 1965). Animal A.H.F. products obtainable as porcine or bovine concentrate are very potent sources of factor VIII (Bidwell, 1955a, 1955b) but are species-specific and produce serious immune reactions in the recipient, of ten after as little as 10 days' administration. Sensitization, once so achieved, may limit the further use of this product, but cross-sensitivity to the other animal product probably seldom occurs. Sensitivity reactions to the human concentrate are minor and non-fatal, and they are usually readily controlled by the oral administration of antihistamine drugs or, if these fail, intravenous hydrocortisone therapy. The human product can, however, transmit homologous serum jaundice.

The present series of cases show that if haemostasis is rendered normal in haemophiliacs suffering from suspected intracranial haemorrhage the usual methods of investigation can then be used to confirm the diagnosis, and particularly to exclude infection as a cause of the clinical signs, and that it is then possible to deal effectively with any surgical problem on its merits. Thus carotid angiography was carried out in this series on seven occasions (in five, human A.H.F. concentrates were used, and in two, animal A.H.F.) with no complications from bleeding. Other authors have been understandably reluctant to undertake this procedure when adequate factor VIII replacement therapy has not been possible ; in one of Simpson and Robson's (1960) patients the procedure probably resulted in the development of a mediastinal haematoma which contributed to the patient's death. J. Potter (personal communication, 1964) reports the successful use of the procedure on two occasions, and Silverstein (1960) and Kerr (1964) once each.

The mortality rates from intracranial bleeding in haemophilioid states reported from other centres vary considerably. Silverstein (1960) gives it as $70 \%$ in his series in which the diagnostic criteria were strictly fulfilled, whereas Kerr (1964) gives an overall mortality of $33 \%$; however, not all of his cases satisfied strict criteria for the diagnosis of intracranial bleeding. Potter (personal communication, 1964), using A.H.F. concentrates in treatment, reported a mortality of $14 \%$.

One patient (Case 5) in our series died. He had survived a series of traumatic procedures, which included craniotomy, but eventually succumbed not to his haemophilia but to intracranial infection. The mortality in this small series was therefore $20 \%$. It is clear, however, that where a satisfactory A.H.F. concentrate is available in sufficient supply intracranial haemorrhage in classical haemophiliacs can now be dealt with on its merits, provided skilled laboratory control of treatment can ensure the maintenance of a normal haemostatic mechanism. The management of such patients should therefore include in successive stages: (a) the establishment of the precise diagnosis of haemophilia and its degree of severity, (b) adequate factor VIII replacement therapy in the patient (which should be maintained for at least 10 days or until wound healing is well established), and $(c)$ the performance of any special investigations which involve trauma to the patient.

The absence of overt trauma as a cause or association of intracranial bleeding in two of these patients (Cases 2 and 4), in neither of whom an arterial lesion was demonstrated, is interesting. One of them (Case 2) has essential hypertension. We have noted that minor trauma, often unnoticed at the time, frequently precedes apparent spontaneous bleeding in haemophiliacs by a few days, and that this is so even in the less severely effected. It would seem probable that this antecedent trauma uses up, at the site of the injury, the limited factor VIII reserve of the patient, and this predisposes him to "spontaneous" haemorrhage, perhaps as a result of the occult trauma elsewhere.

\section{Summary}

Five patients suffering from classical haemophilia (factor VIII deficiency) were admitted to two hospitals with proved intracranial haemorrhage during a five-year period. The four cases admitted to one hospital constituted $3.4 \%$ of all haemophilic admissions for all forms of haemorrhage during that five-year 
period. Of the five patients studied one had a severe degree of haemophilia, in two it was moderate, and in two mild.

The mortality rate in the series was $20 \%$, as one patient eventually died of intracranial infection.

Carotid angiography was safely performed on seven occasions in four of the patients, human A.H.F. concentrate being used on five occasions and porcine A.H.F. concentrate on two.

It is suggested that the administration of these A.H.F. concentrates in sustained and adequate amounts to patients with classical haemophilia who are thought to be suffering from intracranial haemorrhage will possibly make it safe to use the standard techniques in establishing the precise diagnosis, and subsequently to treat a lesion by either conservative or surgical means.

We wish to thank our neurosurgical colleagues Mr. P. Harris, Mr. J. F. Shaw, and Mr. G. N. N. Reddy; and also Professor Sir
John Bruce and Dr. A. Brownlie Smith for their help with some general surgical and otorhinolaryngological aspects respectively of Case 5. We are particularly grateful to Dr. A. S. Brown, consultant anaesthetist, and Dr. A. A. Donaldson, consultant radiologist, for their skilled assistance, in particular in the performance of the percutaneous angiograms.

\section{REFERENCES}

Astrup, T. (1956). Blood, 11, 781.

Bidwell, E. (1955a). Brit. ₹. Haemat., 1, 35.

- (1955b). Ibid., 1, 386.

Biggs, R. (1957). Lancet, 2, 311.
and Douglas, A. S. (1953). 7. clin. Path., 6, 23.

- Eveling, J., and Richards, G. (1955). Brit. ł. Haemat., 1, 20.

and Macfarlane, R. G. (1962). Human Blood Coagulation and its Disorders, 3rd ed., p. 73 . Oxford.

Cumming, R. A., Davies, S. H., Ellis, D., and Grant, W. (1965). Vox Sang (Basel), 10, 687.

Kerr, C. B. (1964). \%. Neurol. Neurosurg. Psychiat., 27, 166.

Silverstein. A. (1960). Arch. Neurol. Psychiat. (Chic.), 3, 141.

Simpson, D. A., and Robson, H. N. (1960). Aust. N.Z.' 7. Surg., 29, 287.

\title{
Indications and Effects of Exchange Transfusion in Adults in Gynaecology and Obstetrics
}

\author{
R. H. PHILPOTT,* M.в., CH.B., M.R.C.o.g. ; N. E. G. FOSTER, $\nmid$ M.в., CH.B. \\ DERK CRICHTON, $\ddagger$ M.B., D.PHI., F.R.C.S., F.R.C.o.G.
}

Brit. med. F., 1966, 2, 1630-1633

Our findings regarding the value and safety of exchange transfusion are reported in this contribution, which follows our preliminary communication on the subject (Philpott, Foster, and Crichton, 1963). Our investigation now includes a sufficient number of cases to justify the substitution of facts for the impressions previously presented.

\section{Nature of Investigation}

This investigation, which extended from 1 November 1962 to 31 October 1963 , included all patients whose haemoglobin level was below 4.4 g. $/ 100 \mathrm{ml}$. (30\%), who did not manifest evidence of shock, and who had been admitted to the gynaecological and obstetric units at King Edward VIII Hospital, Durban.

All cases were investigated and treated by us, and an unbiased comparison of the response to exchange transfusion (group A) as opposed to slow packed-cell transfusion (group B) was undertaken. Bias in selection of either form of treatment was avoided by employing random sampling to dictate the form of treatment.

Method of Exchange Transfusion (Group A).-Blood infusion was made into a forearm vein by means of a No. 15 gauge needle. Plastic bag-containers were used for this transfusion, and a Fenwal pressure-bag was placed around the plastic bag of blood in readiness to pump the blood in under pressure. Each bag of blood was weighed and $1,250 \mathrm{ml}$. of fairly fresh blood in the form of packed cells was used. Blood removal was performed through the femoral vein by means of a bloodrecipient set and vacuum blood-bottles. The rate of withdrawal was controlled by a clamp on the plastic tubing between the

* Senior Lecturer, Head of the Sub-Department of Obstetrics, University of Natal.

† Lecturer, University of Natal.

$\ddagger$ Professor, and Head of the Division of Gynaecology and Obstetrics, University of Natal. needle and the bottle. The procedure began with the withdrawal of $200 \mathrm{ml}$. of blood from the femoral vein. Thereafter blood was pumped in as fast as possible with the Fenwal pump and blood removed at the same rate, so that at the end of the procedure $1,450 \mathrm{ml}$. of blood had been removed and $1,250 \mathrm{ml}$. of packed cells given. Great care is required to ensure that the rate of transfusion and that of removal are equated throughout, and consequently a record of the pulse, blood-pressure, and respiratory rate was kept every five minutes.

Method of Transfusing Packed Cells (Group B).-The rate of transfusion in group B was "tailored" to the clinical condition and response of the patient. Thus, if an increase occurred in the pulse rate, respiratory rate, or jugular venous pressure, then the drip rate was slowed down or stopped until the condition improved. In general the time taken for transfusing 4 units of packed cells was about three days.

Investigations.-Special investigations, undertaken in every case, included the Hess test, bleeding-time, clotting-time, a full blood count (including platelet count), fibrinogen, electrolytes and $\mathrm{CO}_{2}$-combining power, blood urea, calcium, proteins, and liver-function tests. Particular care was taken to exclude cardiac failure in every case. The tests were conducted before transfusion, and in the case of patients in group A they were repeated at 1-hour, 4-hour, and 24-hour intervals after the exchange transfusion. In group $B$ they were repeated daily for three days.

Material.-The series of 50 cases comprised every case admitted to the professorial obstetrical department and gynaecological ward. There were 25 cases in each group. The average age was 25.7 years, ranging from 21 years to 42 . The type of anaemia was diagnosed by full blood count, blood smear, and bone-marrow study. Twenty-seven cases were due to blood loss, 10 to megaloblastic anaemia, and 13 to iron deficiency. The conditions associated with the anaemia are listed in Table I. Evidence of cardiac failure was based 